\title{
Nivel de seguridad percibida por el paciente de hemodiálisis crónica
}

\author{
Verónica Martín Guerrero, Gema Palacios García, Cristina Grano de Oro Hidalgo, Violeta Hernández de Arribas, \\ Jacoba Rodríguez Peña
}

Unidad de Hemodiálisis. Hospital Universitario Ramón y Cajal. Madrid. España

\section{Resumen}

Introducción: Son múltiples las publicaciones que abordan los riesgos hospitalarios, sin embargo escasas las investigaciones que estudian eventos adversos en las unidades de diálisis desde la percepción de los pacientes.

Objetivo: Evaluar el nivel de seguridad percibido por el paciente durante la sesión hemodiálisis.

Material y métodos: Estudio descriptivo, donde participaron 44 pacientes. Al no encontrar ninguna escala validada se autodiseño una a partir de 3 escalas validadas que miden calidad, satisfacción y factores de estrés, elaborando un instrumento de 40 ítems.

Resultados: Se realizó un análisis descriptivo y estratificado por edad y años en hemodiálisis. El $100 \%$ de los encuestados se sienten seguros durante la sesión de hemodiálisis. En relación con la calidad de los cuidados y la percepción de los mismos todos los ítems consiguen una puntuación superior al tres sobre cinco. En cuanto a la educación un $63 \%$ le gustaría recibir más educación aunque un 70\% afirma saber actuar ante las complicaciones. En el listado de factores de estrés, obtienen puntuaciones mayores las complicaciones del acceso vascular, el tiempo conectado a la máquina, el transporte y temperatura de la sala.

Conclusión: La calidad de los cuidados enfermeros percibidos obtiene una buena satisfacción. En cuanto a los factores estresantes creemos que se podría llevar a cabo intervenciones enfermeras para disminuirlos, rea-

\section{Correspondencia:}

Verónica Martín Guerrero

Ctra. de Colmenar Viejo, km. 9,100.28034 Madrid

E-mail:vmartinguerrero@hotmail.com lizando actividades educativas y lúdicas intradiálisis, todo ello para aumentar la seguridad, y la calidad de los cuidados.

PALABRAS CLAVE

- UNIDADES DE HEMODIÁLISIS EN HOSPITAL

- SEGURIDAD

- CALIDAD DE LA ATENCIÓN DE SALUD

- EDUCACIÓNY DIÁLISIS RENAL

Perceived safety level by the patient on chronic hemodialysis

\section{Abstract}

Introduction: There are multiple publications addressing hospital risks, however, manuscripts studying adverse events in dialysis units from the perception of patients are scarce.

Objective: To assess the perceived safety level by the patient during hemodialysis session.

Material and Methods: A descriptive study in which 44 patients participated. Not finding in the literature any validated scale, a scale from 3 others validated, which measured the quality, satisfaction and stress factors, was elaborated, obtaining an instrument of 40 items.

Results: A descriptive analysis was performed and stratified by age and years on hemodialysis. $100 \%$ of respondents feel safe during hemodialysis session. Regarding to the quality of care and the perception of them, all items get a superior three out of five score. With respect to education, $63 \%$ of patients would like more education while $70 \%$ claimed to know to act before 
complications. In the list of stressors, they get higher scores on vascular access complications, time connected to the machine, transportation and room temperature.

Conclusion: The perceived quality of nursing care gets a good satisfaction. With respect to the obtained stressors, we believe that nursing interventions could carry out to reduce them, conducting intradialytic educational and recreational activities with the aim of increasing safety and quality of care.

\section{KEYWORDS}

- HOSPITAL HEMODIALYSIS UNITS

- SAFETY

- QUALITY OF HEALTH CARE

- EDUCATION AND KIDNEY DIALYSIS

\section{Introducción}

Según el Informe de diálisis y trasplante en el año 2014, del total de enfermos renales en terapia renal sustitutiva, la modalidad de hemodiálisis era utilizada por un $79,24 \%$ de los pacientes, en diálisis peritoneal un $16.5 \%$ del total y fueron trasplantados un $4,29 \%$.

La Organización Mundial de la Salud (OMS) lanzó en 2004 la "Alianza Mundial para la Seguridad del Paciente", promoviendo acciones, herramientas y recomendaciones para mejorar la seguridad en todos los países del mundo².

La mejora de la calidad asistencial ha sido siempre inherente a los principios del Sistema Nacional de Salud (SNS), como se desprende de la Ley General de Sanidad en el Título 1, incluye como una de las actuaciones a desarrollar por las administraciones sanitarias la de controlar y mejorar la calidad de la asistencia sanitaria en todos sus niveles. La seguridad del paciente, dimensión esencial en la calidad asistencial, implica desarrollar estrategias para reducir el daño innecesario del paciente asociado a la asistencia sanitaria. Los objetivos de la estrategia han estado básicamente orientados a mejorar la cultura de la seguridad, la formación de los profesionales y la implicación de los pacientes. En el documento sobre Estrategia de seguridad del paciente del SNS para el período 2015-2020, se desarrollan 6 líneas estratégicas se seguridad. En este trabajo nos centraremos en la línea estratégica 4: Participación de los pacientes y ciudadanos en su seguridad. Cuyos objetivos específicos son los de promover que el paciente reciba información de su proceso, formación sobre su enfermedad e involucrarse participando activamente en la misma.

Existen todavía muchas reticencias tanto por parte de los gestores como por parte de los profesionales, que dificultan la implicación de los pacientes en la toma de decisiones y la participación del ciudadano a nivel colectivo en las decisiones sanitarias. A pesar de ello, si es cierto que el rol del paciente está cambiando y esto requiere de un cambio cultural importante en la relación personal sanitario-paciente, basado en la toma de decisiones compartidas. Diferentes estudios epidemiológicos publicados desde los años 90 han contribuido de forma importante al conocimiento de la magnitud, impacto y características de los eventos adversos ligados a la atención sanitaria. No obstante parece que estas cifras también podrían estar infraestimadas porque se basan en datos registrados por los profesionales ${ }^{3}$.

Según la OMS en lo que respecta a la participación de los pacientes y otros usuarios, la iniciativa "Pacientes por su propia seguridad", tiene por objetivo establecer una red mundial de pacientes y organizaciones de pacientes dirigida por ellos mismos para defender su seguridad a escala regional y nacional. El primer taller organizado en el marco de la iniciativa Pacientes por su propia seguridad se celebró en Londres en noviembre de 2005, el cual reunió a pacientes y promotores de la seguridad en la atención que se les dispensa. En 2006 y 2007 se organizaron talleres de seguimiento en regiones de la $0 \mathrm{MS}^{4}$.

En las investigaciones publicadas en los últimos años se puede diferenciar entre estudios realizados con el objetivo de analizar y evaluar la incidencia de errores médicos o efectos adversos, y trabajos que intentan indagar en las opiniones y expectativas de los pacientes y profesionales sobre la seguridad asistencial ${ }^{5}$.

El ciudadano, como paciente y como usuario, está cada vez más preparado para desempeñar un papel más activo en relación con el cuidado de su salud y para aprovechar todas las oportunidades que las nuevas tecnologías le ofrecen. Estamos ante un nuevo tiempo en el que médicos y pacientes deben comprometerse y trabajar conjuntamente, tanto para promover y proteger la salud, como para procurar un uso adecuado de los recursos del sistema sanitario ${ }^{6}$.

Son múltiples las publicaciones que abordan los riesgos hospitalarios, sin embargo, son escasas las investigaciones que estudian los eventos adversos en las unidades 
de diálisis, así como aquellas que estudian estos aspectos desde la percepción de los pacientes ${ }^{7}$.

La Enfermedad Renal Crónica (ERC) es una de las enfermedades que presenta mayores niveles de impacto psicosocial en los pacientes que la sufren. La combinación de numerosos factores de estrés agudos y crónicos hacen del paciente con ERC un sujeto con altos niveles de sufrimiento físico y psíquico ${ }^{8}$.

Los pacientes con enfermedad renal terminal necesitan tratamiento para poder mantenerse con vida. La mayoría de estos pacientes se encuentran en tratamiento renal sustitutivo en hemodiálisis, y según varios estudios, una gran parte de estos pacientes se sienten inseguros durante su tratamiento. Debido a la naturaleza crónica de la enfermedad, la sensación de inseguridad durante la sesión puede influir en la calidad de vida del paciente9.

El concepto de calidad de vida relacionada con la salud (CVRS) aparece en las sociedades occidentales en un momento en que la esperanza de vida ha aumentado y en el que el papel de la medicina no debe ser únicamente el de proporcionar muchos años de vida a las personas, sino, sobre todo, el de aportar una mejora en la calidad de los años vividos. La CVRS es un concepto unipersonal, multideterminado (actitud frente a la vida, creencias, actividad laboral, etc.) y que no siempre coincide con las estimaciones realizadas por el médico. Las personas que padecen una enfermedad renal crónica suelen estar más interesadas en conseguir la mejor calidad de vida. Los pacientes deben someterse a tratamiento sustitutivos altamente invasivos que involucran cambios de vida en los ámbitos: físico, psicológico y social para el paciente y su familia. Para las personas en hemodiálisis, la diálisis se convierte en el eje de su vida, implicando grandes restricciones ${ }^{10}$.

El ingreso de un paciente en un programa de diálisis periódica, supone un punto de inflexión importante en su vida y en muchas ocasiones marca un antes y un después. Provocando una explosión de sentimientos que sumados al deficiente estado de salud, pueden generar actitudes que llegan a condicionar la evolución de la enfermedad. Entre los principales factores desencadenantes de estrés estaría: miedo a la muerte, miedo a "la máquina", síntomas físicos que no desaparecen con el tratamiento o que aparecen como consecuencias del mismo (mareos, hipotensiones, cansancio fácil, disminución de la líbido, impotencia), incertidumbre sobre el trasplante, limitaciones en la vida social y laboral, dieta restrictiva y alteración de la autoimagen provocada por el acceso vascular ${ }^{11}$.
El riesgo a provocar un daño en el paciente aumenta a medida que sus cuidados se vuelven más complejos. La hemodiálisis es un factor de riesgo para la salud de los pacientes sometidos a este tipo de tratamiento. Durante la hemodiálisis, una máquina sustituye en parte la función renal mediante la circulación de la sangre del paciente a través de un riñón artificial. Varios aspectos del tratamiento provocan que sea complejo, por ejemplo, el uso de equipo técnico avanzado y el riesgo asociado a infecciones vasculares. Para reducir los riesgos de hemodiálisis se han llevado a cabo diversas iniciativas, como innovaciones para mejorar la calidad del agua o reducir las infecciones del acceso vascular. Sin embargo, no se ha prestado mucha atención a las respuestas emocionales de los pacientes, ni a la percepción de seguridad de estos, que podría ser diferente a la percibida por el sistema de salud?.

Varios autores han investigado sobre la seguridad desde el punto de vista del paciente. Garrick ${ }^{11}$, describió como la mitad de los pacientes en hemodiálisis sentían preocupación por que sucediera un error durante su tratamiento, siendo mayor que la que el personal podría predecir. Feroeze ${ }^{13}$, mostró en su estudió que la mitad de los pacientes experimentaban ansiedad durante el tratamiento. En ambos estudios las situaciones que provocan un aumento de la ansiedad fueron: una nueva o desconocida enfermera y las alarmas de la máquina. Lovink ${ }^{9}$, llego a la conclusión en su estudio de que los pacientes eran conscientes de los muchos riesgos de su tratamiento, pero la enfermera tuvo un papel fundamental en la sensación de seguridad de los pacientes en hemodiálisis, por lo que la mayoría de ellos expresaron sensación de seguridad durante su tratamiento.

Dada la escasa bibliografía encontrada respecto a la percepción de seguridad del paciente en una sala de hemodiálisis y al importante papel que la enfermería puede desarrollar para conseguirlo; vemos necesario realizar un estudio para conocer en qué aspectos el paciente refiere mayor inseguridad, y así poder llevar a cabo si fuera necesario, futuras intervenciones que aumenten su sensación de seguridad, las cuales hayan podido pasar desapercibidas en protocolos de seguridad, al tratarse estos generalmente desde un punto de vista más técnico.

Como objetivo principal del estudio se formuló evaluar el nivel de seguridad percibido por el paciente durante la sesión hemodiálisis.

Como objetivos secundarios se evaluó el nivel de conocimientos del paciente respecto a su patología y 
la capacidad de actuación del paciente frente a las complicaciones derivadas del tratamiento, conocer el nivel de comunicación que percibe el paciente con respecto al personal de enfermería, evaluar el nivel de calidad percibida por el paciente durante la sesión de hemodiálisis e identificar los principales factores de estrés para el paciente durante su tratamiento de hemodiálisis.

\section{Material y métodos}

\section{Diseño}

Se trata de un estudio descriptivo transversal observacional. Realizado en la unidad de hemodiálisis del Hospital Universitario Ramón y Cajal.

\section{Población de estudio y muestra}

La población de estudio fueron los pacientes en tratamiento de hemodiálisis en el Hospital Universitario Ramón y Cajal. Se partió de una población total susceptible de estudio de 46 pacientes en turnos de mañana y tarde.

\section{Criterios de inclusión}

Los criterios de inclusión fueron ser mayor de edad, llevar en hemodiálisis en nuestro servicio más de tres meses y aceptar voluntariamente la participación en el estudio.

\section{Criterios de exclusión}

Los criterios de exclusión fueron no tener capacidades cognitivas suficientes para responder al cuestionario. Declinar la participación.

Tras la aplicación de los criterios anteriores se obtuvo una muestra de 44 pacientes, tras la exclusión de un paciente por capacidades cognitivas insuficientes para responder al cuestionario y otro por fallecimiento.

\section{Variables de estudio}

Las variables de estudio sociodemográficas y clínicas fueron: edad, sexo, tiempo en hemodiálisis, persona con la que vive, quién se encarga de la alimentación, nivel de estudios y deambulación.

Las variables de resultados fueron: calidad de los cuidados enfermeros prestados percibidos por el paciente, conocimiento y capacidad de actuación del paciente ante las complicaciones, seguridad percibida respecto al tratamiento y factores de estrés vinculados al tratamiento identificados por el paciente.

\section{Instrumento de medida}

Tras una búsqueda de la literatura publicada relacionada con la seguridad del paciente en general y en concreto del paciente en hemodiálisis no se ha encontrado ninguna encuesta validada que mida el objetivo de este estudio, la percepción de seguridad del paciente en una sala de hemodiálisis. Por ello se elabora un cuestionario a partir de las encuestas:

- Encuesta de calidad de cuidados de enfermería (CUCACE) ${ }^{13}$, versión en castellano del NSNS (The Newcastle satisfaction with Nursing Scales). Utilizamos la sección 2 del mismo, consta de 19 preguntas sobre la opinión del paciente en relación a la atención de las enfermeras. Se trata de un cuestionario con escala tipo Likert, del 1 (Nada satisfecho) y el 5 (Completamente satisfecho). De este cuestionario utilizamos 5 preguntas que creemos son las que más se adaptan a nuestro tipo de estudio, ya que el resto están relacionadas con la hospitalización.

- Encuesta SERVQHOS ${ }^{14}$, instrumento que nos permite evaluar la calidad percibida por el paciente, utilizada en estudios de satisfacción de los usuarios de Servicios Sanitarios, que combina expectativas y percepciones. Se trata de un cuestionario ya validado, que ha demostrado una alta consistencia interna, una buena capacidad predictiva, y especialmente una buena estructura factorial, ha demostrado discriminar entre pacientes satisfechos e insatisfechos y además nos permite identificar oportunidades de mejora. Se trata de puntuar 19 ítems, cada uno puntúa con escala Likert, desde 1 (mucho peor de lo que esperaba) hasta 5 (mucho mejor de lo que esperaba). De dicho cuestionario se seleccionan 6 preguntas que son las que mejor se adaptan al objeto de estudio, relacionando calidad con seguridad.

- Conocimientos, seguridad y capacidad de actuación ante las complicaciones, se elabora un instrumento basándonos en el Manual de Protocolos y Procedimientos de Actuación de Enfermería Nefrológica, editado por la SEDEN ${ }^{15,16}$. Con 16 preguntas sobre educación y seguridad del paciente.

- Listado de factores de estrés ${ }^{17,18,19}$, para medir este grupo de variables, se ha utilizado el cuestionario realizado por Barba en su Tesis doctoral: el enfermo renal crónico: análisis de su experiencia psicosocial en la hemodiálisis hospitalaria, diseño de esta escala es similar al empleado por otros autores, como Baldree, en su Haemodialysis Stressor Scale (HSS). Consistente en una lista de 16 items cerrados y uno abierto de los principales factores de estrés que se pueden dar en el proceso de diálisis y en sus consecuencias, elegidas 
en función de la investigación de Barba (1998) y de las aportaciones realizadas por personal sanitario especializado en este tipo de patología y su tratamiento. El paciente ha de indicar si cada uno de los elementos citados les genera o no estrés, malestar físico y $/ 0$ psíquico. En caso afirmativo debe puntuar el grado de estrés provocado en una escala de 1 a 10, en la que una mayor puntuación supone un mayor nivel de malestar. Utilizamos 12 preguntas que creemos que son las que más se adaptan a nuestro tipo de estudio

Finalmente teniendo en cuenta lo descrito, se autodiseña un instrumento compuesto por 40 ítems, con tres tipos de respuesta, abierta, cerrada y de escala likert de 5 puntos.

Los 11 primeros ítems evalúan la calidad de los cuidados, los 6 ítems siguientes el conocimiento y la capacidad, del ítems 18 al 27 la seguridad y los últimos 12 ítems los factores de estrés vinculados, quedando un último ítems abierto para alguna observación que quiera añadir el paciente. Ver anexo con la encuesta autodiseñada.

\section{Recogida de datos}

Las encuestas fueron autocumplimentadas por los pacientes, durante la sesión de diálisis, a partir de la primera hora de conexión y una vez que el paciente estaba estable.

Se le invitó a participar a todos los pacientes que cumplían los criterios de inclusión, se le explicaba en que consistía la encuesta y se solicitaba su consentimiento por escrito en caso de querer participar.

En el caso de que el paciente no la pudiera rellenar, bien porque la fístula se encontrara en su brazo dominante 0 porque refiriera problemas para leer la encuesta, esta fue heterocumplimentada por un profesional, para evitar posibles sesgos al contestarla, se instruyó a una enfermera no perteneciente al servicio, con la cual se consensuó la manera de administrar el cuestionario para que todos los pacientes respondieran de forma correcta a las preguntas.

Todas las encuestas fueron administradas el mismo día a todos los pacientes del turno. Empleando un total de dos días (dos mañanas y dos tardes).

\section{Consideraciones éticas}

Previo a la cumplimentación de la encuesta, todos los participantes del estudio fueron informados de forma clara en que consistía el mismo y manifestaron su consentimiento de formar parte del estudio por escrito firmando el correspondiente documento de consentimiento de manera voluntaria. La información obtenida fue confidencial y tratada conforme a la Ley Orgánica de Datos de Carácter Personal 15/99.

\section{Análisis estadístico}

Las variables cualitativas se expresan como frecuencias y porcentajes. Las variables cuantitativas como medias y desviaciones típicas, si cumplen criterios de normalidad y medianas para las asimétricas.

Se efectúo un análisis estratificado por edad (mayor y menor de 65 años) y tiempo de tratamiento en hemodiálisis (menor de 1 año o mayor de 3 años) y la variable sociodemográfica dependencia con la pregunta acerca de la ayuda para acomodarse en la sala. Los datos se analizaron con el paquete estadístico SPSS, V15.0.

\section{Resultados}

En el presente estudio se recogieron un total de 44 cuestionarios pertenecientes a pacientes en hemodiálisis crónica del Hospital Ramón y Cajal.

En relación a la edad la mediana fue de $71,5 \pm 16,12$, siendo la edad máxima de los pacientes 91 años y la mínima 27 años. En relación al género 30 pacientes, un $68,2 \%$ eran varones y 14 pacientes, un $31,8 \%$ mujeres.

Respecto al tipo de convivencia de los pacientes, el $45,5 \%$ de los casos ( $n=20$ ) vive con su pareja, el 29,5 $\%(n=13)$ sola/o, el $9,1 \%(n=4)$ con sus hijos, un $4,5 \%$ $(n=2)$ en instituciones y un $11 \%(n=5)$ en otras circunstancias.

En cuanto a la persona que se encarga de la alimentación de 17 pacientes $(38,6 \%)$ se ocupa la pareja, de 16 pacientes $(36,4 \%)$ se ocupan ellos mismos, de 3 pacientes $(6,8 \%)$ se encarga la institución y de 8 $(18,2 \%)$ otras personas.

El nivel de estudio de los participantes fue de 16 de los casos $(36,4 \%)$ sin estudios, 11 de los casos (25\%) formación media y 17 de los casos $(38,6 \%)$ estudios superiores.

En relación al tiempo en hemodiálisis 7 pacientes $(15,9 \%)$ llevan menos de un año, 12 pacientes $(27,3 \%)$ entre uno y tres años y 25 pacientes $(56,8 \%)$ más de tres años.

Del total, un $50 \%$ de la muestra $(n=22)$ son independientes, un $38,6 \%$ de la muestra $(n=17)$ necesitan 
ayuda, un $2,3 \%$ de la muestra $(n=1)$ es independiente en silla de rueda y un $9,1 \%$ de la muestra $(n=4)$ son dependientes.

En relación con las respuestas a la primera parte de la encuesta relacionada con la calidad de los cuidados y la percepción de los mismos por parte del paciente, destacamos que todos los ítems consiguen una puntuación superior al tres sobre cinco puntos. Las mayores puntuaciones corresponden con la presencia de una enfermera cuando se la necesitaba y a la frecuencia con la que la enfermera comprobaba su estado. Y las peores puntuaciones están relacionadas con la tecnología de los equipos y la información médica dada a los familiares. Ver tabla 1. hemodiálisis, un $97 \%$ asegura que el personal contesta de forma clara y comprensible a sus dudas.

En cuanto a la educación un $63 \%$ le gustaría recibir más educación sobre sus cuidados y en relación a los conocimientos más de un $70 \%$ saben actuar ante las complicaciones más frecuentes preguntadas, destacando que un $43 \%$ no sabrían actuar en el caso de parada de la FAV.

En el análisis segmentado destacar que sólo un 55,6\% de los mayores de 65 años conocen la importancia de comprimirse el punto de punción tras la retirada de las agujas frente a un $70,6 \%$ de los menores de 65 años. En cuanto al ítems de si existe algún momento de la

Tabla 1. Puntuación media por ítem que valora la calidad de cuidados y calidad percibida.

\begin{tabular}{|c|c|c|c|c|}
\hline & VÁLIDOS & PERDIDOS & MEDIA & $\begin{array}{l}\text { DESV. } \\
\text { TÍPICA }\end{array}$ \\
\hline Presencia de la enfermera & 44 & 0 & 4,18 & 0,843 \\
\hline Información de la enfermería & 44 & 0 & 3,77 & 0,831 \\
\hline Comprobación de enfermería de sus estado & 44 & 0 & 4,23 & 0,803 \\
\hline Conciencia de enfermería de sus necesidades & 44 & 0 & 3,77 & 0,886 \\
\hline \multirow[t]{2}{*}{ Intimidad proporcionada por enfermería } & 44 & 0 & 3,61 & 0,895 \\
\hline & VÁLIDOS & PERDIDOS & MEDIA & $\begin{array}{c}\text { DESV. } \\
\text { TÍPICA }\end{array}$ \\
\hline Satisfacción con tecnología & 44 & 0 & 3,45 & 0,730 \\
\hline Rapidez de atención & 44 & 0 & 3,52 & 0,876 \\
\hline Confianza transmitida por personal & 43 & 1 & 3,84 & 0,814 \\
\hline Amabilidad de trato del personal & 44 & 0 & 3,86 & 0,852 \\
\hline Satisfacción con trato personalizado & 44 & 0 & 3,73 & 0,817 \\
\hline Información médica a familiares & 44 & 0 & 3,23 & 1,008 \\
\hline
\end{tabular}

Al realizar el análisis segmentado por tiempo en diálisis y edad de los pacientes se observan datos similares en todos los ítems, destacando que la valoración de la presencia de una enfermera cerca sigue siendo la más valorada en todos los casos mayor de 4,13 puntos. Por otra parte la mayor diferencia observada es al valorar la rapidez con la que consiguen lo que necesitan, siendo los pacientes con menor tiempo en diálisis los que dan mejor puntuación 4 puntos frente a los que llevan más de tres años con una puntuación de 3,15.

La segunda parte de la encuesta que valoraba los conocimientos, seguridad y capacidad de actuación ante las posibles complicaciones, señalamos que el $100 \%$ de los encuestados se sientes seguros durante la sesión de sesión que le provoque más inseguridad, los pacientes mayores de 65 años identifican una mayor número de momentos inseguros $44,4 \%$, frente a los menores de 65 años que sólo un 17,6\% refieren algún momento de inseguridad.

Los mayores momentos de inseguridad a los que hacen referencia los pacientes durante la sesión de hemodiálisis son la hipotensión en la última hora de la sesión y los pinchazos

Sólo la mitad de los pacientes menores de 65 años consideran el uso del ecógrafo como un elemento de seguridad frente al $70,6 \%$ de los pacientes mayores de 65 años. 
La tercera parte de la encuesta con un listado de factores de estrés, los que obtuvieron puntuaciones mayores fueron el funcionamiento correcto del acceso vascular $(6,61)$, tiempo conectado a la máquina $(6,48)$, transporte sanitario $(6,18)$, temperatura de la sala $(6,09)$, y por último la presencia de una nueva enfermera $(5,18)$. Por otro lado los factores que menos estrés provocan fueron la relación con el personal de enfermería $(2,93)$ y los familiares $(1,75)$. Ver tabla 2.
En el análisis segmentado los pacientes con menos de un año en hemodiálisis puntúan de modo diferentes los siguientes factores de estrés, iluminación $(4,57)$, tiempo $(7,29)$ y nueva enfermera $(3,86)$ frente a los que llevan más de tres años que puntúan con 2,33, 5,83 y 5,46 respectivamente. $Y$ por otro lado los menores de 65 años valoran la autonomía $(5,47)$, la postura $(5,88)$ y las complicaciones $(6,82)$ frente a los mayores de 65 años con $3,74,4,22$ y 3,59 respectivamente.

Tabla 2. Puntuación media por ítem que valora los factores estresantes.

\begin{tabular}{|c|c|c|c|c|}
\hline & VÁLIDOS & PERDIDOS & MEDIA & $\begin{array}{l}\text { DESV. } \\
\text { TÍPICA }\end{array}$ \\
\hline Autonomía & 44 & 0 & 4,41 & 3,259 \\
\hline Enfermedad & 44 & 0 & 2,93 & 2,546 \\
\hline Tratamiento & 44 & 0 & 3,07 & 2,757 \\
\hline Cuidados & 44 & 0 & 2,84 & 2,605 \\
\hline Temperatura & 44 & 0 & 6,09 & 3,079 \\
\hline Iluminación & 44 & 0 & 3,07 & 2,889 \\
\hline Ruido & 44 & 0 & 2,84 & 2,641 \\
\hline Médicos & 44 & 0 & 2,57 & 2,618 \\
\hline Enfermeras & 44 & 0 & 1,84 & 2,112 \\
\hline Familiares & 44 & 0 & 1,75 & 1,966 \\
\hline Postura & 44 & 0 & 4,86 & 2,914 \\
\hline Transporte & 44 & 0 & 6,18 & 4,036 \\
\hline Tiempo & 44 & 0 & 6,48 & 2,510 \\
\hline Dolor & 44 & 0 & 4,30 & 2,962 \\
\hline Nueva enfermedad & 44 & 0 & 5,18 & 3,244 \\
\hline Complicaciones & 44 & 0 & 4,84 & 3,184 \\
\hline Alarma & 44 & 0 & 2,59 & 2,083 \\
\hline Acceso & 44 & 0 & 6,61 & 2,652 \\
\hline
\end{tabular}

Al estratificar la dependencia con la ayuda recibida en la sala de hemodiálisis un $41 \%$ de los pacientes que necesitan ayuda no consideran recibirla correctamente.

\section{Discusión}

En este estudio partimos de la premisa de que los pacientes podrían sentir poca seguridad durante la sesión de hemodiálisis, sin embargo un total de los sujetos estudiados se sienten seguros durante su tratamiento de hemodiálisis en nuestro hospital, destacando que las situaciones que más temor provocan durante el tratamiento son las hipotensiones en la última hora y los pinchazos.
En relación a la calidad de los cuidados enfermeros percibidos por el paciente se obtienen una buena satisfacción coincidiendo con los resultados encontrados en la bibliografía. Hernández, en su estudio refleja datos similares en todos los ítems, coincidiendo tanto en las puntuaciones mejor valoradas como en las peor valoradas, la confianza con el personal donde obtuvo un 3,82 y nuestro estudio un 3,84 como mejor puntuados en ambos estudios y por otro lado como peor puntuación fue la relacionada con la tecnología de los equipos con un 3,52 y 3,45 respectivamente.

En cuanto a los conocimientos que los pacientes tienen, aunque un alto porcentaje afirma tener información suficiente ha quedado de manifiesto que le gustaría recibir una mayor educación sobre sus cuidados. 
Los factores estresantes con mayor puntuación coinciden con los de la literatura consultada, Barba destaca en su artículo coincidiendo con nuestros resultados, que los factores que más estrés provocan son los relacionados con el procedimiento de hemodiálisis en sí, seguido de los factores ambientales y la dependencia que la enfermedad genera debido al tratamiento.

La principal limitación de este estudio es que aunque se recoge al total de la población accesible, sería interesante ampliar el conocimiento con mayor número de pacientes pertenecientes a otros centros, dado que esto podría dar una mayor validez externa a los resultados obtenidos.

En segundo lugar, los datos obtenidos pueden estar sesgados al haberse realizado las encuestas dentro del centro sanitario en el que el paciente recibe el tratamiento, aunque se intentó aminorar este sesgo de información, siendo la persona encargada de administrar la encuesta alguien ajeno a la unidad. Otra limitación fue que no se encontró ninguna escala validada con las características de estudio. Sería interesante contar con escalas validadas que se ajusten a las características y necesidades del enfermo renal en hemodiálisis.

Tras el estudio de los datos obtenidos y su análisis, se concluye que para mejorar la seguridad percibida por el paciente en la sala de diálisis, consideramos que deberíamos llevar a cabo intervenciones enfermeras que refuercen los conocimientos en relación a los accesos vasculares y educación sobre los cuidados en general. En relación a la duración del tratamiento sugerimos llevar a cabo actividades intradiálisis relacionadas con el ocio y entretenimiento para paliar esta percepción.

\section{Agradecimientos}

Nos gustaría dar las gracias a los pacientes que participaron en el estudio y al Servicio de Nefrología de Hospital Ramón y Cajal. Una mención especial a la Supervisora de Investigación de Enfermería Ana Rodríguez por su tiempo y dedicación.

Recibido: 20 abril 16

Revisado: 6 junio 16

Modificado: 24 junio 16

Aceptado: 4 julio 16

\section{Bibliografía}

1. Registro Español de Enfermos Renales. Informe de diálisis y trasplante 2014. XLV Congreso Nacional de la Sociedad Española de Nefrología; 2014. http://www.senefro.org/contents/webstructure/REER_Cong_SEN_2015.pdf (último acceso 10 marzo 2016).

2. World Health Organization (WHO). World Alliance for Patient Safety: Forward Programme 2005 [monografía en internet]. Geneva (Switzerland): WHO; October 2004. [Citado 18 Feb de 2016]. Disponible en: http://www.who.int/patientsafety.

3. Estrategia de Seguridad del Sistema Nacional de Salud. Período 2015-2020. Sanidad 2015. Ministerio de Sanidad, Servicios Sociales e Igualdad. [Citado 18 Feb de 2016].Disponible en: http:// www.seguridaddelpaciente.es/.../Estrategia\%20 Seguridad\%20del\%20Paciente\%202015-2020. pdf.

4. Organización Mundial para la Salud. 59a Asamblea Mundial de la Salud. 2006. Punto 11.16 del orden del día provisional. [Citado 18 Feb de 2016].Disponible en: http://apps.who.int/gb/archive/pdf_files/WHA59/A59_22-sp.pdf.

5. La perspectiva de los ciudadanos por la seguridad del paciente. Informes, estudios e investigación 2011. Ministerio de Sanidad, Política Social e Igualdad. [Citado 18 Feb de 2016].Disponible en: www.msssi.gob.es/organizacion/.../Perspectiva_ciudadanos_SP.pdf.

6. Aibar RC. La percepción del riesgo: del paciente informado al paciente consecuente. Humanitas: Humanidades médicas. 2005. [Citado 18 Feb de 2016].Disponible en: www.fundacionmhm.org/ pdf/Mono8/Articulos/articul03.pdf.

7. Hernández M M, Ochango GA, Royuela GC, Lorenzo MS, Paniagua F. Seguridad clínica percibida por los pacientes en diálisis peritoneal. Rev Soc Esp Enferm Nefrol. 2008; 11 (3):190-195.

8. Barba TA, Pérez RE, Gómez GY, Barba ST, Machi PM. La evaluación del estrés en la Insuficiencia Renal Crónica: una aproximación multidisciplinar. Rev Soc Esp Enferm Nefrol. 1998;1(1):22-26.

9. Lovink MH, Kars MC, de Man-Van Ginkel JM, Schoonhoven L. Patients'experiences of safety during haemodialysis treatmente a qualitative 
study. Journal of Advanced Nursing.2015;71(10): 2374-2383.

10. Cobo SJ et al. Factores sociológicos y calidad de vida relacionada con la salud en pacientes en hemodiálisis. Rev Soc Esp Enferm Nefrol.2011;14(2):98-104.

11. Amador CR, Pons RE, Espinosa CC. Depresión y ansiedad en pacientes en hemodiálisis: la creatividad para combatirlas. Comunicaciones Presentadas al XXXIV Congreso Nacional SEDEN. Rev Soc Esp Enferm Nefrol.2009. [Citado 18 Feb de 2016]. Disponible en: www.revistaseden.org/files/2126_ Páginas\%20de\%202009-11.pdf.

12. Garrick R, Kliger A, Beth S. Patient and facility safety in hemodiálisis: opportunities and strategies to develop a culture of safety. Clin J Am Soc Nephol.2012;7(abril):680-688.

13. Feroze $U$ et al. Anxiety and depresión in maintenance diálisis patients: preliminary data of a cross-sectional study and brief literature review. Journal of Renal Nutrition. 2012;22(1):207-210.

14. Alonso R, Blanco-Ramos MA, Gayoso P. Validación de un cuestionario de calidad de cuidados de enfermería. Rev Calidad Asistencial. 2005;20(5):246250.

15. Borré OY, Lenis VC, González RG. Utilidad del cuestionario SERVQHOS-E para medir calidad percibida de la atención de enfermería. CES Salud Pública.2014;5(2):127-136.
16. Miranda GM. et al. Protocolo de atención de enfermería a pacientes en tratamiento con hemodiálisis. En Miranda GM. (eds.) Manual de protocolos y procedimientos de atuación en enfermería nefrológica. $1^{\text {a }}$ ed. Madrid: Grupo E.Entheos. S.L; 2001. 39-158.

17. Heung M, Adamowki T, Segal JH y Malani PN. A sucessful approach to fall prevention in an outpatient hemodiálisis center. Clin J Am Soc Nephrol.2010:5;1775-1779.

18. Barba TA. El enfermo renal crónico: análisis de su experiencia psicosocial en la hemodiálisis hospitalaria. Tesis doctoral. Universidad de Salamanca; 2014.

19. Barba AA. La evaluación del estrés en la insuficiencia renal crónica: una aproximación multidisciplinar. Enfermería Nefrológica.1998;1:22-26.

20. Baldre KS, Murphy SP y Powers MJ. Stress identification and coping patterns in patients on haemodialyisis. Nursing Research.1982;31(2):107-112.

21. Anguera, E. y Martínez, F. Estudio multicéntrico sobre la calidad asistencial percibida por los pacientes en programa de hemodiálisis en Tarragona. Rev Soc Enferm Nefrol. 2011;14(3):172-180. 